\title{
Maximum Liquefaction of Gases Revisited
}

\author{
R. Pitanga Marques ${ }^{1 *}$, P.R.G. Bordoni ${ }^{2}$, M. O. Pinho ${ }^{3}$ \\ ${ }^{1}$ Department of Mechanical Engineering, CEFET-RJ \\ ${ }^{2}$ Department of Computer Science, Universidade Federal do Rio de Janeiro (UFRJ) \\ ${ }^{3}$ Department of Mathematics, CEFET-RJ \\ E-mail: ${ }^{1}$ rupitanga@hotmail.com
}

Received 6 September 2021, Revised 11 December 2021, Accepted 25 January 2022

\begin{abstract}
Joule-Thomson liquefiers are the commonest machines to liquefy gases. Over the years, countless number of articles have been published on the subject. Dozens of $1^{\text {st }}$ and $2^{\text {nd }}$ law analyses were carried out on Joule-Thomson liquefaction cycles. And yet an aspect of purely theoretical interest seems to have passed unnoticed, namely: for a given volume of gas, what conditions should be fulfilled to achieve maximum liquefaction without considering engineering details of design equipment and the highly irreversible character of work-consuming devices, heat exchangers, heat leaks and the throttling process. This work addressed this issue by applying the $1^{\text {st }}$ law analysis and elementary calculus prescriptions to a simple Linde-Hampson liquefying process. The same approach could be applied to other liquefying cycles. As is well-known, for a given mass flow rate of a gas, maximum fraction liquefied occurs when the pre-cooling temperature, $T_{i}$, and initial pressure, $P_{i}$, lie on the inversion curve. It has been proved that this is only true if an additional condition is fulfilled. Expressions for it were derived for the van der Waals, RKS and PR equations of state.
\end{abstract}

Keywords: Liquefaction of gases; Joule-Thomson effect; maximum fraction liquefied; inversion curves; liquefier.

\section{Introduction}

Commercial liquefaction of gases is a century-old technology. The pioneering work of Carl von Linde in Germany, William Hampson in England and Georges Claude in France opened a new branch of science and technology: cryogenics [1]. From biological research, where liquid nitrogen is used to freeze blood cells, tissues, and similar, to space flight, where liquid oxygen is used in combination with liquid hydrogen to propel rockets, liquefied gases have found a variety of applications in modern industrial societies [2].

In throttling processes, fluids flow through a restriction which can be an orifice, a valve or a porous plug. They undergo, in general, an increase or a decrease in temperature. The Joule-Thomson (or Kelvin) coefficient is a measure of the change in temperature which results from a drop in pressure across the constriction. And is defined by

$\mu=\left(\frac{\partial T}{\partial P}\right)_{h}$

A throttling process does not necessarily entail cooling or heating. An isenthalpic expansion of ideal gases results in no temperature change. From this point of view, the JouleThomson coefficient can be seen as a measure of departure from ideal-gas behaviour.

Joule-Thomson coefficients may be positive, zero (as in the case of ideal gases) or negative. Fig. 1 below illustrates, $\mu$, for different gases as a function of the temperature.

For most real gases, the Joule-Thomson coefficient gives rise to a decrease of temperature within only a certain domain of temperature and pressure. At room temperatures (see Fig.
1), the coefficient is positive for nearly all gases. Thus, if a gas undergoes an isothermal compression, and is allowed to expand to low pressures, part of it will be liquefied. The higher the pressure or lower the temperature, the higher the non-ideality is to produce enough cooling to liquefy the gas [4].

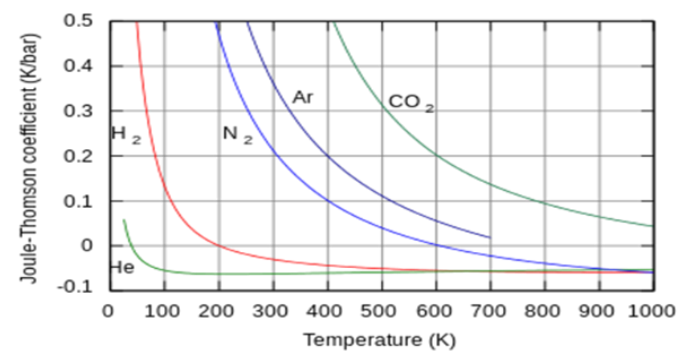

Figure 1. Joule-Thomson coefficients as a function of the temperature for different gases [3].

Most liquefaction cycles use the Joule-Thomson effect to attain a certain level of non-ideality in the cryogenic gas. In the Linde-Hampson liquefier, a nozzle is used to produce the Joule-Thomson effect to cool the gas to become a liquid. The incoming gas is compressed in a multistage compressor to high pressure before delivery to the cooler, which is a recuperative heat exchanger. This counter-flow heat exchanger cools down the incoming gas to a temperature below the inversion temperature by exchanging heat with chilled water available. The cold gas is constantly recycled to cool more incoming compressed gas. Because of the cumulative cooling effect, the gas gradually becomes cold enough until liquefaction occurs after expansion at the 
throttle. In the Claude liquefier, part of the gas from the multistage compressor goes to the expander where it does work. The expansion engine operates adiabatically to lower the temperature of part of the high pressure gas. The work of the expander partially compensates for the work of compression and reduces the requirement for very high pressures in the liquifier. The gas cooled down in the process is used to cool the incoming compressed gas in the cooler. As in the Linde liquefier, the continuing flow of gas will decrease the temperature at a point where part of the gas will be liquefied by the Joule-Thomson effect. For gases with a negative Joule-Thomson coefficient, near-standard conditions (hydrogen, helium, neon), the Claude should be replaced by the Collins liquefier - an extension of the Claude liquefaction process with a sophisticated mechanical design of reciprocating expanders and heat exchangers. This is because the cooling duty required is the highest among cryogens for the removal of the sensible heat. The incoming gas from the compressor is cooled by liquid nitrogen in the cooler. For a detailed exposition of all cryogenic liquefaction processes and liquefiers, the interested reader should consult Mukhopadhyay [5].

The performance of liquefiers can be assessed by means of the concept of the figure of merit (FOM). A very good description of FOM and the thermodynamics associated with cryogenic plants may be found in references $[6,7]$. FOM is defined by the ratio of the ideal work, $-W_{i}$ for a thermodynamically ideal liquefaction process to the actual work required to liquify the cryogenic gas, $-W_{l}$. Thus,

$$
F O M=\frac{-W_{i}}{-W_{l}}
$$

Table.1 illustrates the performance of a simple LindeHampson liquefier for different gases.

Table 1. Work of liquefaction and FOM of a simple LindeHampson process ${ }^{*}$ for different cryogens(reproduced under permission from Mukhopadhyay [4]) $* P_{1}=1 \mathrm{~atm}, P_{2}=200$ atm, $T_{1}=T_{2}=300 \mathrm{~K}, 100 \%$ efficiency of isothermal compressor and $100 \%$ effectiveness of the main heat exchanger with no temperature difference between the incoming and outgoing streams at the inlet.

\begin{tabular}{lcccc}
\hline Cryogen & $\begin{array}{l}\text { Normal } \\
\text { boiling } \\
\text { point } \\
(\mathrm{K})\end{array}$ & $\begin{array}{c}\% \\
\text { Liquefied }\end{array}$ & $\begin{array}{l}\text { Work of } \\
\text { liquefaction }\end{array}$ & FOM \\
& 77.4 & 7.08 & 6673 & 0.115 \\
$\mathrm{~N}_{2}$ & 78.8 & 8.08 & 5621 & 0.131 \\
$\mathrm{Air}$ & 87.3 & 11.83 & 2750 & 0.174 \\
$\mathrm{Ar}$ & 90.2 & 10.65 & 3804 & 0.167 \\
$\mathrm{O}_{2}$ & 111.7 & 19.77 & 3957 & 0.276 \\
$\mathrm{CH}_{4}$ & 184.5 & 52.57 & 611 & 0.588 \\
$\mathrm{C}_{2} \mathrm{H}_{6}$ & \multicolumn{5}{|c}{} \\
\hline
\end{tabular}

Like all cyclic machines, liquefiers depend on components performance to maximise the liquid yield. The highly irreversible character of work-consuming devices, heat exchangers, heat leaks and the throttling process do reduce the fraction liquefied. In a very interesting study, B.Z. Maytal [8] showed that for any real Linde-Hampson machine of finite size, the recuperator can be optimized to reach extreme rates of performance. For a similar group of liquefiers an optimal flow rate was found to maximize the rate of production of liquid cryogen. A study by M. Kanoglu et al [9] based on $1^{\text {st }}$ and $2^{\text {nd }}$ law analyses of a simple Linde-
Hampson cycle established the minimum work requirement, applicable to any cryogen to enhance the performance of the liquefier. More recently, C. Yilmaz et al. [10] carried out a comprehensive thermodynamic analysis of the simple LindeHampson, precooled Linde-Hampson, Claude, and Kapitza cycles. They were model in the computer environment and analyzed with Engineering Equation Solver (EES) software program. The authors concluded that the Claude cycle delivers the largest liquid yield while the Kapitza cycle, the best exergy efficiency. Nontheless, they pondered that despite their low efficiencies, the simple Linde-Hampson and precooled Linde-Hampson cycles offer the simplicity of their setup.

To the best of the authors' knowledge, the only work found in the literature related to this was published by A. T. A. M. de Waele [11]. The author carried out a $1^{\text {st }}$ law analysis of a simple Linde-Hampson machine and obtained, as expected, the expression for the liquefied fraction exactly the same as ours. However, de Waele did not investigate the (thermodynamic) pre-requisites for it to achieve maximum liquefaction.

This work aims to discuss a question raised by Zemansky and Dittman [12] on the optimal theoretical conditions to liquefy common gases.

\section{Thermodynamic Model}

Consider the steady-state liquefying system shown pictorially in Fig. 2 and corresponding T-s diagram .

For simplicity, engineering details of the system components are omitted. The objective is to determine the maximum fraction liquefied from first principals. Zemansky and Dittman's analysis captures enough of the physics of liquefaction by identifying the states visited by the stream of gas. This paper followed Zemansky and Dittman's reasoning and kept their notation.

At steady state, liquid is formed at a constant rate: a certain mass flow rate fraction, $\boldsymbol{y}$, is liquefied and stored in a vessel for later use, and $1-\boldsymbol{y}$ of mass flow rate fraction of low-pressure gas pre-cools the incoming high-pressure gas in a counter-flow heat exchanger. A fresh stream of makeup gas at room temperature joins the low-pressure stream of gas before entering the compressor to replenish the cycle. Considering there are no pressure drops, no heat leaks, no temperature of approach at the warm end of the heat exchanger, a simple first law analysis yields

$h_{i}=\boldsymbol{y} h_{L}+(1-\boldsymbol{y}) h_{f}$.

This shows the enthalpy of the incoming gas equals the enthalpy of $\boldsymbol{y}$ units of mass flow rate in liquid form plus the enthalpy of $1-\boldsymbol{y}$ units of mass flow rate of the outgoing gas. Or:

$\boldsymbol{y}=\frac{h_{f}-h_{i}}{h_{f}-h_{L}}$

Where:

$\boldsymbol{h}_{\boldsymbol{i}}$ is the enthalpy of the incoming gas at $\left(T_{i}, P_{i}\right)$

$\boldsymbol{h}_{\boldsymbol{L}}$ is the enthalpy of the fraction liquefied at $\left(T_{L}, P_{L}\right)$

$\boldsymbol{h}_{\boldsymbol{f}}$ is the enthalpy of the outgoing gas at $\left(T_{f}, P_{f}\right)$

if the heat exchanger and throttling valve are thermally insulated (schematically shown by the dotted lines in the figure). 


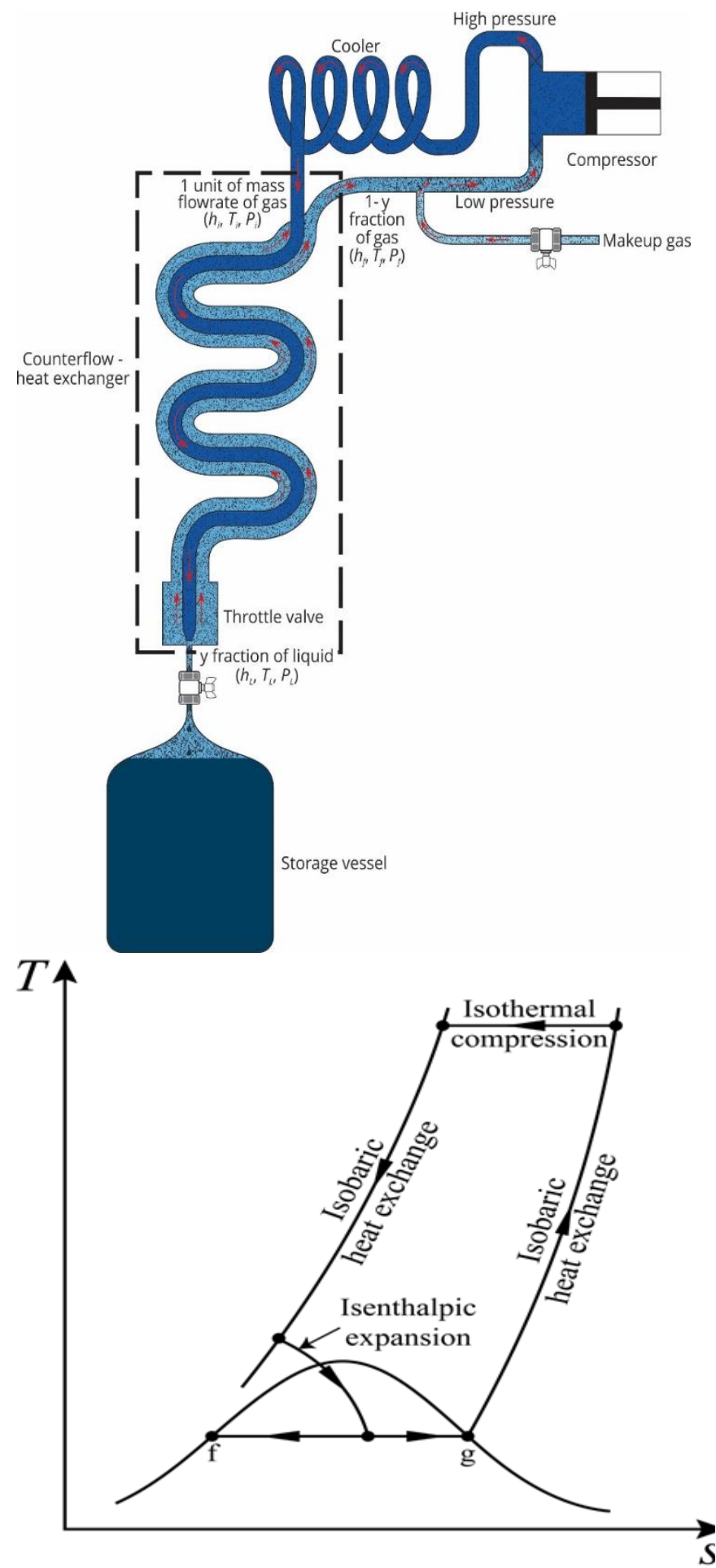

Figure 2. Pictorial view of a simple Linde-Hampson liquefier (inspired on a figure of the book of Zemansky and Dittman [12]) and correspondingT-s diagram drawn by Mrs. Isabel Menezes.

The enthalpy of the incoming gas, $\boldsymbol{h}_{\boldsymbol{i}}$, is a function of pressure that may be chosen at will (the optimum pressure to start throttling corresponds to a point on the inversion curve) at a fixed $T_{i} ; \boldsymbol{h}_{\boldsymbol{L}}$, the enthalpy of the fraction liquefied at the entry of the storage vessel, is independent of both pressure and temperature, and therefore, constant. $\boldsymbol{h}_{\boldsymbol{f}}$, the enthalpy of the outgoing gas, despite the pressure drop in the return pipe, remains nearly constant. Therefore, the fraction liquefied, $\boldsymbol{y}$, is a function of, $\boldsymbol{h}_{\boldsymbol{i}}$, only. For maximum liquefaction (highest possible value of $\boldsymbol{y}$ ), $\boldsymbol{h}_{\boldsymbol{i}}$ ought to be a minimum, i.e.: $\left(\frac{\partial h_{i}}{\partial P}\right)_{T=T_{i}}=-\left(\frac{\partial h_{i}}{\partial T}\right)_{P}\left(\frac{\partial T}{\partial P}\right)_{h_{i}}=-c_{p} \mu=0$

Zemansky and Dittman posited on page 284 of their book that $\boldsymbol{y}$ to be a maximum, $\mu=0$ at $T=T_{i}$ since $c_{p}$ cannot be zero except at the absolute zero. But they did not carry their analysis any further and promptly concluded that "...the point $\left(T_{i}, P_{i}\right)$ must lie on the inversion curve in order to maximize the fraction $\boldsymbol{y}$ of the liquid ".

Elementary calculus shows that after establishing the critical points of a function by setting $f^{\prime}(x)=0$, we need to calculate the second order derivative to determine whether such points are a local maximum or a local minimum. If the function $f(x)$ is twice differentiable at a critical point, $x$, then:

if $f^{\prime \prime}(x)<0$ then $f(x)$ has a local maximum at $x$.

if $f^{\prime \prime}(x)=0$ then the test is inconclusive.

if $f^{\prime \prime}(x)>0$ then $f(x)$ has a local minimum at $x$.

In brief, the sign of the second order derivative should be studied, $\left(\frac{\partial^{2} h_{i}}{\partial P^{2}}\right)_{T}$, in order to decide whether the point $\left(T_{i}, P_{i}\right)$ lies on the inversion curve.

\subsection{The Joule-Thomson Inversion Curve}

The locus of all points at which the Joule-Thomson coefficient, $\mu$, is zero is called the inversion curve, shown schematically in red in Fig.3 (a P-T diagram in reduced coordinates, $T_{r}=T / T_{c}$ and $P_{r}=P / P_{c}$ ). The locus is a collection of points of maximum values of curves of equal enthalpy. The inversion curve joins together points at which the slope $\left(\frac{\partial T}{\partial P}\right)_{H}$ changes from positive to negative according to elementary calculus prescriptions $\left(\frac{\partial T}{\partial P}\right)_{h}=0$ and $\left(\frac{\partial^{2} T}{\partial P^{2}}\right)_{h}<0$. The inversion curve, which is parabolic in shape, is a boundary between two regions: the region of cooling where $\mu>0$, and the region of heating where $\mu<$ 0 . The upper part cuts the $\mathrm{T}$-axis at the maximum inversion temperature while the lower part ends, abruptly, at the vapour pressure curve (Fig.3) [13-14]. An isobar drawn on the diagram intersects several isenthalps (shown in black) at a number of points at which $\mu$ is calculated by measuring the slope.

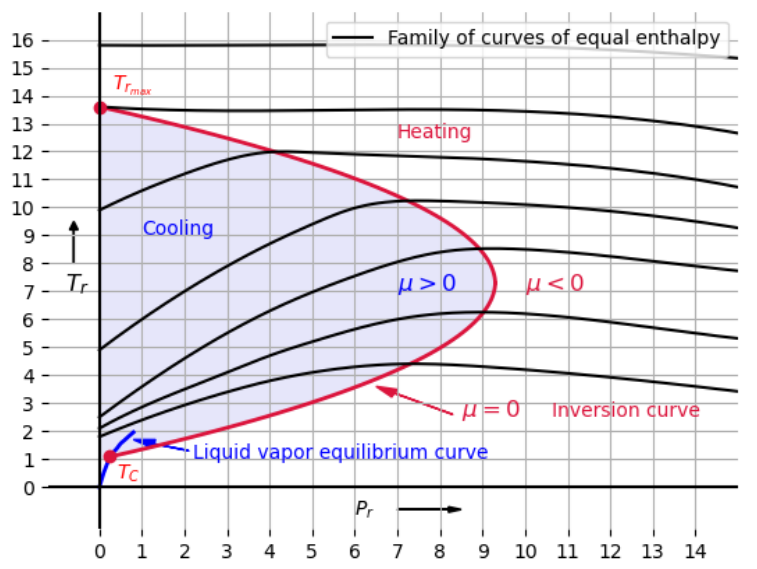

Figure 3. Joule-Thomson inversion curve drawn by one of the authors. 
To draw the inversion curve, experimental points for which $\mu=0$ are required. Since volumetric data of sufficient accuracy for gases are rare at highly reduced temperatures, the upper part is either determined by a leastsquare fit or by an equation of state (EoS) [13].

Over the years, several authors have calculated the JouleThomson inversion curve by making use of equations of state, [13-22]. The calculation of the Joule-Thomson inversion curve for each new EoS provides a severe test for its predictive capability. A better agreement with experimental inversion curves of different substances is a clear indication of its superiority.

Written in reduced coordinates, the predictive inversion curve (for an equation of state) is given by,

$T_{r}\left(\frac{\partial P_{r}}{\partial T_{r}}\right)_{V_{r}}+V_{r}\left(\frac{\partial P_{r}}{\partial V_{r}}\right)_{T_{r}}=0$

A plot of an inversion curve on a P-T plane in reduced temperature and pressure should yield a curve that is valid for all gases. This behaviour can only be justified by evoking the theorem of corresponding states: chemical compounds at equal reduced pressures and temperatures have equal reduced volumes. Strictly speaking, the theorem of corresponding states is only valid for the so-called simple fluids; those whose force field has a high degree of symmetry. Fortunately, most liquefied gases $\left(\mathrm{Ar}, \mathrm{CH}_{4}, \mathrm{~N}_{2}\right.$, $\mathrm{O}_{2}, \mathrm{C}_{2} \mathrm{H}_{4}$ etc.) fall into this category. Next, the derivatives $\left(\frac{\partial P_{r}}{\partial T_{r}}\right)_{V_{r}}$ and $\left(\frac{\partial P_{r}}{\partial V_{r}}\right)_{T_{r}}$ can be determined from an arbitrarily chosen equation of state. A judicious choice depends, to a large extent, on which class of fluids the equation of state was designed for. Van der Waals equation of state ( $\mathrm{vdW}$ EoS), despite its severe limitations, offers a useful picture of the thermodynamic properties for simple fluids. And unlike modern cubic equations of state, it is far more superior (e.g. Redlich-Kwong-Soave (RKS) EoS [23], Peng-Robinson (PR) EoS [24 ] and RK-PR EoS [25]), vdW EoS

$P=\frac{R T}{V-b}-\frac{a}{V^{2}}$

obeys the theorem of corresponding states. Written in reduced form,

$\left(P_{r}+\frac{3}{V_{r}^{2}}\right)\left(3 V_{r}-1\right)=8 T_{r}$,

van der Waals equation applies in principle to any substance, but can only provide actual properties when critical properties are known.

Inserting the derivatives $\left(\frac{\partial P_{r}}{\partial T_{r}}\right)_{V_{r}}$ and $\left(\frac{\partial P_{r}}{\partial V_{r}}\right)_{T_{r}}$ of (8) in (6) yields,

$-\frac{T_{r}}{\left(3 V_{r}-1\right)^{2}}+\frac{3}{4} \frac{1}{V_{r}^{2}}=0$.

A rather involved algebraic manipulation to eliminate $V_{r}$ between (6) and (7) results in the well-known van der Waals inversion curve,

$T_{r}=3\left[1 \pm \frac{\sqrt{9-P_{r}}}{6}\right]^{2}$

which is illustrated in Fig. 4.

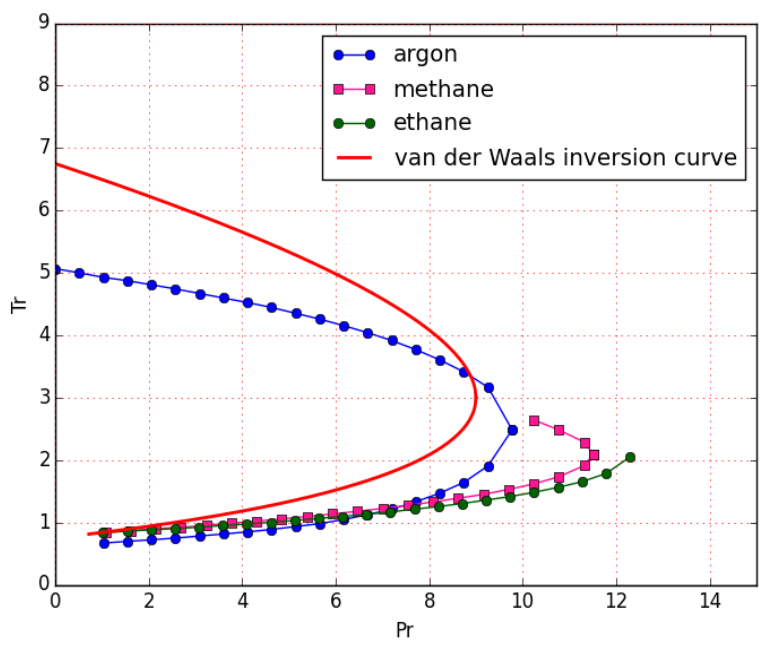

Figure 4. Van der Waals inversion curve and experimental data for three commercial gases (data collected from [26]) drawn by one the authors .

When $\left(\frac{\partial T}{\partial P}\right)_{h}=0$ and $\left(\frac{\partial^{2} T}{\partial P^{2}}\right)_{h}<0$ are applied to vdW EoS, yields,

$T_{i n v}=\frac{2 a}{R b} \frac{(v-b)^{2}}{v^{2}} \cong \frac{2 a}{R b}$

the maximum inversion temperature, $T_{i n v}$, i. e. the highest temperature for which $\mu=\left(\frac{\partial T}{\partial P}\right)_{h}$ is positive so that a reduction in pressure results in a decrease in temperature. Table 2 shows maximum inversion temperature values, boiling points (for comparison) and critical points for common gases.

Table 2. Maximum inversion temperatures, boiling points and critical points for common gases (data collected from [27]).

\begin{tabular}{lccc}
\hline cryogen & $\begin{array}{l}\text { maximum } \\
\text { inversion } \\
\text { temperature, } \\
T_{\text {inv }}(\mathrm{K})\end{array}$ & $\begin{array}{c}\text { Critical } \\
\text { temperature } \\
(\mathrm{K})\end{array}$ & $\begin{array}{c}\text { Normal } \\
\text { boiling } \\
\text { point }(\mathrm{K})\end{array}$ \\
\hline $\mathrm{He}$ & 51 & 5.2 & 4.2 \\
$\mathrm{H}_{2}$ & 205 & 33.2 & 20.4 \\
$\mathrm{Ne}$ & 250 & $44.4 \mathrm{~K}$ & 27.07 \\
$\mathrm{~N}_{2}$ & 621 & 126 & 77.4 \\
$\mathrm{O}_{2}$ & 893 & 154 & 90.2 \\
$\mathrm{Ar}$ & 794 & 150.7 & 87.3 \\
$\mathrm{CH}_{4}$ & 939 & 190.7 & 111.7 \\
\hline
\end{tabular}

\section{Results and Discussion}

To achieve maximum liquefaction, $\boldsymbol{h}_{\boldsymbol{i}}$ ought to be a minimum, i.e.:

$\left(\frac{\partial h_{i}}{\partial P}\right)_{T=T_{i}}=0 \quad$ and $\quad\left(\frac{\partial^{2} h_{i}}{\partial P^{2}}\right)_{T=T_{i}}>0$

Or:

$\left(\frac{\partial\left(-c_{p} \mu\right)}{\partial P^{2}}\right)_{T=T_{i}}>0$ since $\left(\frac{\partial h_{i}}{\partial P}\right)_{T=T_{i}}=-c_{p} \mu$

but $\mu=\frac{1}{c_{P}}\left[T\left(\frac{\partial v}{\partial T}\right)_{P}-v\right]$ or $-c_{p} \mu=\left[v-T\left(\frac{\partial v}{\partial T}\right)_{P}\right]$ 
thus:

$$
\begin{aligned}
& \left(\frac{\partial\left(-c_{p} \mu\right)}{\partial P^{2}}\right)_{T=T_{i}}=\left\{\left(\frac{\partial v}{\partial P}\right)_{T}-\left(\frac{\partial T}{\partial P}\right)_{v}\left(\frac{\partial v}{\partial T}\right)_{P}-\right. \\
& \left.T\left(\frac{\partial^{2} v}{\partial P \partial T}\right)_{T, P}\right\}_{T=T_{i}}
\end{aligned}
$$

and by making use of the cyclic relation:

$\left(\frac{\partial T}{\partial P}\right)_{v}\left(\frac{\partial P}{\partial v}\right)_{T}\left(\frac{\partial v}{\partial T}\right)_{P}=-1$

Eq. (14) becomes:

$\left(\frac{\partial\left(-c_{p} \mu\right)}{\partial P^{2}}\right)_{T=T_{i}}=\left\{2\left(\frac{\partial v}{\partial P}\right)_{T}-T\left(\frac{\partial^{2} v}{\partial P \partial T}\right)_{T, P}\right\}_{T=T_{i}}$

Given the pressure-explicit vdW EoS:

$p=\frac{R T}{(v-b)}-\frac{a}{v^{2}}$

one can easily determine $\left(\frac{\partial v}{\partial P}\right)_{T}$ and $T\left(\frac{\partial^{2} v}{\partial T \partial P}\right)_{P, T}$. These are as follows:

$$
\begin{aligned}
& \left(\frac{\partial v}{\partial P}\right)_{T}=\left[\frac{2 a}{v^{3}}-\frac{R T}{(v-b)^{2}}\right]^{-1} \\
& T\left(\frac{\partial^{2} v}{\partial P \partial T}\right)_{T, P}=\frac{R T}{(v-b)^{2}}\left[\frac{2 a}{v^{3}}-\frac{R T}{(v-b)^{2}}\right]^{-2}
\end{aligned}
$$

And the expression for the second order derivative of the enthalpy with respect to pressure at constant temperature becomes,

$$
\left(\frac{\partial^{2} h_{i}}{\partial P^{2}}\right)_{T}=2\left[\frac{2 a}{v^{3}}-\frac{R T}{(v-b)^{2}}\right]^{-1}-\frac{R T}{(v-b)^{2}}\left[\frac{2 a}{v^{3}}-\frac{R T}{(v-b)^{2}}\right]^{-2}
$$

For $\left(\frac{\partial^{2} h_{i}}{\partial P^{2}}\right)_{T}>0(17)$ can be re-written as:

$\frac{4 a}{v^{3}}-\frac{3 R T}{(v-b)^{2}}>0$

And the solution of the inequality (18) becomes:

$T_{i}<\frac{4 a(v-b)^{2}}{3 R v^{3}}$

Written in reduced coordinates yields:

$T_{i r}<\frac{3}{2} \frac{\left(V_{r}-\frac{1}{3}\right)^{2}}{V_{r}^{3}}$

Expressions for $T_{i}$ obtained from RKS EoS [23] and PR EoS [24] are given in the Appendix.

\section{Conclusions}

Joule-Thomson liquefiers are a suitable means to produce the liquefaction of gases. They are technically much simpler than the multistage cascade liquefiers. And they have two important advantages. Firstly, the lower the temperature, the larger the drop in temperature for a given pressure drop. Secondly, the absence of moving parts requires no lubrication and this is crucial when working at low temperatures. Their efficiency, however, depends on several factors (performing work-consuming devices, effective heat exchangers, absence of heat leaks and the efficiency of the throttling process) to produce the maximum fraction liquefied.

By a simple 1st law analysis and elementary calculus prescriptions (maximum and minimum conditions imposed on functions of one variable) to a simple Linde-Hampson liquefying process, maximum fraction liquefied is obtained when $\boldsymbol{h}_{\boldsymbol{i}}$ is a minimum. From a theoretical point of view, this is achieved when the pre-cooling temperature, $T_{i}$, and initial pressure, $P_{i}$, lie on the inversion curve and $T_{i r}<\frac{9}{4} \frac{\left(V_{r}-\frac{1}{3}\right)^{2}}{V_{r}^{3}}$. As for a more accurate equation of state than of van der Waals', such as RKS or PR EoS, expressions for the second order derivative of $\boldsymbol{h}_{\boldsymbol{i}}$ (given in the Appendix), and therefore $T_{i}$, are algebraically too complicated to draw a conclusive physical picture.

\section{Acknowledgements:}

The authors are grateful to Dr. Martín Cismondi Duarte from the Facultad de Ciencias Exactas Físicas y Naturales of the National University of Córdoba for fruitful discussions on inversion curves from cubic equations of state. The authors would also like to express their gratitude to Mrs. Isabel Menezes for skillfully drawing the Linde-Hampson liquefier and corresponding T-s diagram. The authors are also indebted to Prof. Mamata Mukhopadhyay and the editorial board of PHI Learning Private Ltd. for permission to reproduce table 3.2 on page 51 of her book (Fundamentals of Cryogenic Engineering).

\section{Nomenclature}

a attraction parameter in the van der Waals equation of state $\left[\mathrm{m}^{6} \mathrm{kPa} / \mathrm{kg}^{2}\right]$

$b \quad$ effective molecular volume in the van der Waals

equation of state $\left[\mathrm{m}^{3} / \mathrm{kg}\right]$

$c_{p} \quad$ specific heat capacity $[\mathrm{kJ} / \mathrm{kg} \mathrm{K}]$

EoS equation of state

FOM figure of merit

$h_{i} \quad$ enthalpy of the incoming gas at $\left(T_{i}, P_{i}\right)[\mathrm{kJ} / \mathrm{kg}]$

$h_{L} \quad$ enthalpy of the liquid yield at $\left(T_{L}, P_{L}\right)[\mathrm{kJ} / \mathrm{kg}]$

$h_{f} \quad$ enthalpy of the outgoing gas at $\left(T_{f}, P_{f}\right)[\mathrm{kJ} / \mathrm{kg}]$

$P R \quad$ Peng-Robinson cubic equation of state

$P \quad$ pressure $[\mathrm{kPa}]$

$R \quad$ universal gas constant $[\mathrm{kJ} / \mathrm{kg} \mathrm{K}]$

$R K S \quad$ Redlich-Kwong-Soave cubic equation of state

$R K-P R$ Combined Redlich-Kwong-Soave and PengRobinson equation of state

$T \quad$ temperature $[\mathrm{K}]$

$v d W \quad$ van der Waals cubic equation of state

$v$ molar volume $\left[\mathrm{m}^{3} / \mathrm{kg}\right]$

$y \quad$ fraction of liquefied gas [dimensionless]

Greek letters

$\mu \quad$ Joule-Thomson coefficient $[\mathrm{K} / \mathrm{Pa}]$

$\omega \quad$ acentric factor [dimensionless]

\section{Subscripts}

c critical value

$i \quad$ incoming

$f \quad$ outgoing

L liquid

$r \quad$ reduced property 


\section{Appendix}

For a matter of convenience, the cubic equations of state are written in the following form:

$P=\frac{R T}{V-b}-\frac{\Gamma(T)}{\Psi(V)}$

such that $\Gamma(T) \neq 0$ and $\Psi(V) \neq 0$

For the van der Waals EoS:

$\Gamma(T)=a \quad$ and $\quad \Psi(V)=V^{2}$

For Redlich-Kwong-Soave (RKS) EoS:

$\Gamma(T)=a\left(1+m\left(1-T_{r}^{0.5}\right)\right)^{2}$

where $m=0.480+1.574 \omega-0.176 \omega^{2}$

and $\omega$ is the acentric factor.

$\Psi(V)=V(V-b)$

And for Peng-Robinson (PR) EoS:

$\Gamma(T)=a\left(1+m\left(1-T_{r}^{0.5}\right)\right)^{2}$

where $m=0.37464+1.54226 \omega-0.26992 \omega^{2}$

and $\omega$ is the acentric factor.

$\Psi(V)=V^{2}+2 b V-b^{2}$

Rewriting (A) and making use of the implicit function theorem, yields:

$f(P, T, V)=P-\frac{R T}{V-b}+\frac{\Gamma(T)}{\Psi(V)}$

Then:

$\frac{\partial V}{\partial P}=-\frac{\frac{\partial f}{\partial P}}{\frac{\partial f}{\partial V}} \quad$ and $\quad \frac{\partial V}{\partial T}=-\frac{\frac{\partial f}{\partial T}}{\frac{\partial f}{\partial V}}$

$\frac{\partial f}{\partial V}=\frac{R T}{(V-b)^{2}}-\frac{\Gamma(T)}{\Psi(V)^{2}} \frac{d \Psi}{d V}$

$\frac{\partial f}{\partial P}=1$

$\frac{\partial f}{\partial T}=\frac{R T}{V-b}-\frac{1}{\Psi(V)} \frac{d \Gamma(T)}{d T}$

Inserting the derivatives above in B, one gets:

$\frac{\partial V}{\partial P}=-\frac{1}{\left[\frac{R T}{(V-b)^{2}}-\frac{\Gamma(T) d \Psi}{\Psi(V)^{2} d V}\right]}$

$\frac{\partial V}{\partial T}=\frac{\frac{R}{(V-b)}-\frac{1}{\Psi(V)} \frac{d \Gamma(T)}{d T}}{\left[\frac{R T}{(V-b)^{2}}-\frac{\Gamma(T)}{\Psi(V)^{2}} \frac{d \Psi}{d V}\right]}$

and $\quad \frac{\partial^{2} V}{\partial P \partial T}=0 \quad$ since $\frac{\partial V}{\partial T} \quad$ doesn't depend on P.
As a result:

$\frac{\partial V}{\partial P}=-\frac{1}{\left[\frac{R T}{(V-b)^{2}}-\frac{\Gamma(T) d \Psi}{\Psi(V)^{2} d V}\right]}>0$

$\frac{R T}{(V-b)^{2}}-\frac{\Gamma(T)}{\Psi(V)^{2}} \frac{d \Psi}{d V}<0 \quad$ or $\quad \frac{R T}{(V-b)^{2}}<\frac{\Gamma(T)}{\Psi(V)^{2}} \frac{d \Psi}{d V}$

Finally,

$\frac{T}{\Gamma(T)}<\frac{1}{R}\left(\frac{V-b}{\Psi(V)}\right)^{2} \frac{d \Psi}{d V}$

For vdW EoS , (C) becomes:

$\frac{T}{\mathrm{a}}<\frac{1}{R}\left(\frac{V-b}{V}\right)^{2} \frac{d \Psi}{d V} \quad$ or $\quad \frac{T}{\mathrm{a}}<\frac{1}{R}\left(\frac{V-b}{V}\right)^{2} V^{2}$

$T<\frac{2 a}{R V}\left(\frac{V-b}{V}\right)^{2}$

For RKS EoS:

$\frac{R T}{a\left(1+m\left(1-T_{r}^{0.5}\right)\right)^{2}}<2\left(\frac{V-b}{V(V-b)}\right)^{2}(2 V-b) \quad$ or

$\frac{R T}{a\left(1+m\left(1-T_{r}^{0.5}\right)\right)^{2}}<\frac{a}{R V^{2}}(2 V-b)$.

And for PR EoS:

$\frac{R T}{a\left(1+m\left(1-T_{r}^{0.5}\right)\right)^{2}}<2\left(\frac{V-b}{V^{2}+2 b V-b^{2}}\right)^{2}(V+b)$

$\frac{R T}{a\left(1+m\left(1-T_{r}^{0.5}\right)\right)^{2}}<2 \frac{a}{R}\left(\frac{V-b}{V^{2}+2 b V-b^{2}}\right)^{2}(V+b)$.

\section{References:}

[1] W. Foerg, "History of cryogenics: the epoch of the pioneers from the beginning to the year 1911", Int. J. of Refrigeration 25 283-292. https://doi.org/10.1016/S0140-7007(01)00020-2, 2002.

[2] J. P. Mosquera 2018 "Applications of Cryogenics: Past, Present, Future - Physics World”, Available : https://physicsworld.com/a/applications-of-cryogenicspast-present-future/2018, 2018.

[3 ] Joule-Thomson effect - Wikipedia.

https://pt.wikipedia.org/w/index.php?title=Efeito_JouleThomson\&oldid=56114263, last update 29 August 2019.

[4] C. G. Haseldon, Cryogenics Fundamentals, Academic Press Inc. New York, 1971.

[5] M. Mukhopadhyay, Fundamentals of Cryogenic Engineering, PHI Learning, Eastern Economy Edition, Delhi (India), January 2021.

[6] T. M. Flynn, Cryogenic Engineering, Marcel Dekker, 2005.

[7] R. Barron , Cryogenic Systems, $2^{\text {nd }}$ ed. Oxford Science Publications, Oxord University Press, New York, 1985. 
[8] Kanoglu, M., Dincer, I., Rosen, M.A., "Performance analysis of gas liquefaction cycles", Int. J. of Energy Research 32(1): pp 35 - 43, January 2008

https//:doi.org:10.1002/er.1333

[9] B.-Z. Maytal, "Maximizing production rates of the Linde-Hampson machine", Cryogenics 46 : 49-54, 2006. https://doi:10.1016/j.cryogenics.2005.11.004

[10] C. Yilmaz, T. H. Cetin, B. Ozturkmen, M. Kanoglu "Thermodynamic Performance analysis of gas liquefaction cycles for cryogenic applications" $J$. of Thermal Engineering, (5, (1): pp. 62-75, January 2019. https://doi.org/10.18186/thermal.513038

[11] A. T. A. M. de Waele, "Basics of Joule-Thomson Liquefaction and JT Cooling", J. of Low Temp Phys 186:385-403, 2017. https://doi 10.1007/s10909-0161733-3

[12] M. W. Zemansky, R. H. Dittman, Heat and Thermodynamics: an Intermediate Textbook, 7th ed. New York, McGraw-Hill Companies Inc., pp. 282-284, 1997.

[13] G. D. Miller, "Joule-Thomson inversion curve, corresponding states, and simpler equations of state", Ind. Eng. Chem. Fundamentals 9: 585-589, 1970.

[14] R. D. Gunn, P. L. Chueh , J. M. Prausnitz, "Inversion temperatures and pressures for cryogenic gases and their mixtures", Cryogenics 6: 324-329, 1966.

[15] K. Juris, L. A. Wenzel, "A study of inversion curves", AIChE Journal 18: 684-68, 1972. https://doi.org/ 10.1002/aic.690180404

[16] W. G. Dilay, A. R. Heidemann, "Calculation of JouleThomson inversion curves from equations of state", Industrial Eng. Chem. Fundamentals 25: 158-164, 1986. https://doi.org/ 10.1021/i100021a024

[17] D. Geana , V. Feroiu, "Calculation of Joule-Thomson inversion curves from a general cubic equation of state",
Fluid Phase Equilibria 77: 121-132, 1992. https//:doi.org/10.1016/0378-3812 (92)85100-M.

[18] A. Maghari, S. Matin, "Prediction of Joule-Thomson inversion curves from van der Waals type equations of state", J. of Chem. Eng. of Japan 30: 520-525, 1997. https://doi.org/10.1252/jcej.30.520

[19] C. M. Colina, C. Olivera-Fuentes, "Prediction of the Joule-Thomson inversion curve of air from cubic equations of state". Cryogenics 38: 721-728, 1998. https//: doi.org/10.1016/S0011-2275(98)00036-8

[20] N. S. Matin, B. Haghighi, "Calculation of JouleThomson inversion curves from cubic equations of state". Fluid Phase Equilibria 175: 273-284, 2000. https//: doi.org/10.1016/S0378-3812(00)00443-X

[21] B. Haghighi, M. R. Laee , N. S. Matin, “A comparison among five equations of state in predicting the inversion curves of some fluids", Cryogenics 43: 393-398, 2003.

[22] B. Haghighi , M. R. Bozorgmehr, "Joule-Thomson inversion curves calculation by using equation of state", Asian J. of Chemistry 24: 533-537, 2002.

[23] G. S. Soave, "Equilibrium constants from a modified Redlich-Kwong equation of state", Chemical Eng. Science 27: 1197-1203, 1972.

[24] D. -Y Peng, D. B. Robinson, “ A new two constant equation of state", Ind. Eng. Chem. Fundamen. vol 15, pp. 59-64, 1976.

[25] M. D. Cismondi, J. Mollerup, "Development and application of a three parameter RK-PR equation of state", Fluid Phase Equilibria 232: 74-89, 2005. https//: doi.org/10.1016/j.fluid.2005.03.020.

[26] P. E. Liley, R. C. Reid, E. Buck, " Physical and Chemical Data in : Perry, R. H. and Green D., Perry's Chemical Engineers' Handbook', 7th Ed. McGraw-Hill Companies Inc., Singapore, pp. 3-109, 3-110, 1984.

[27] D. Elwell and A. J. Pointon, Classical Thermodynamics, $1^{s t} E d$. Peguin Library of Physical Sciences, p.175, 1972. 